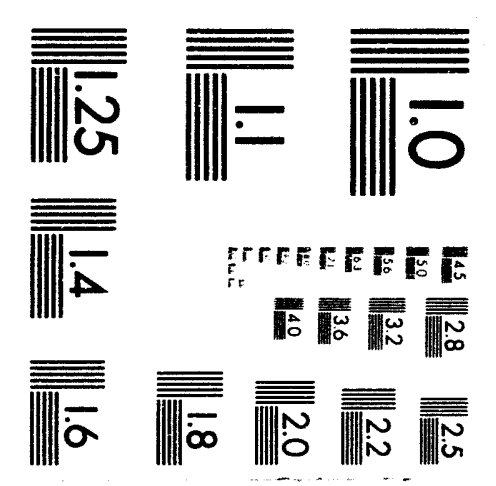



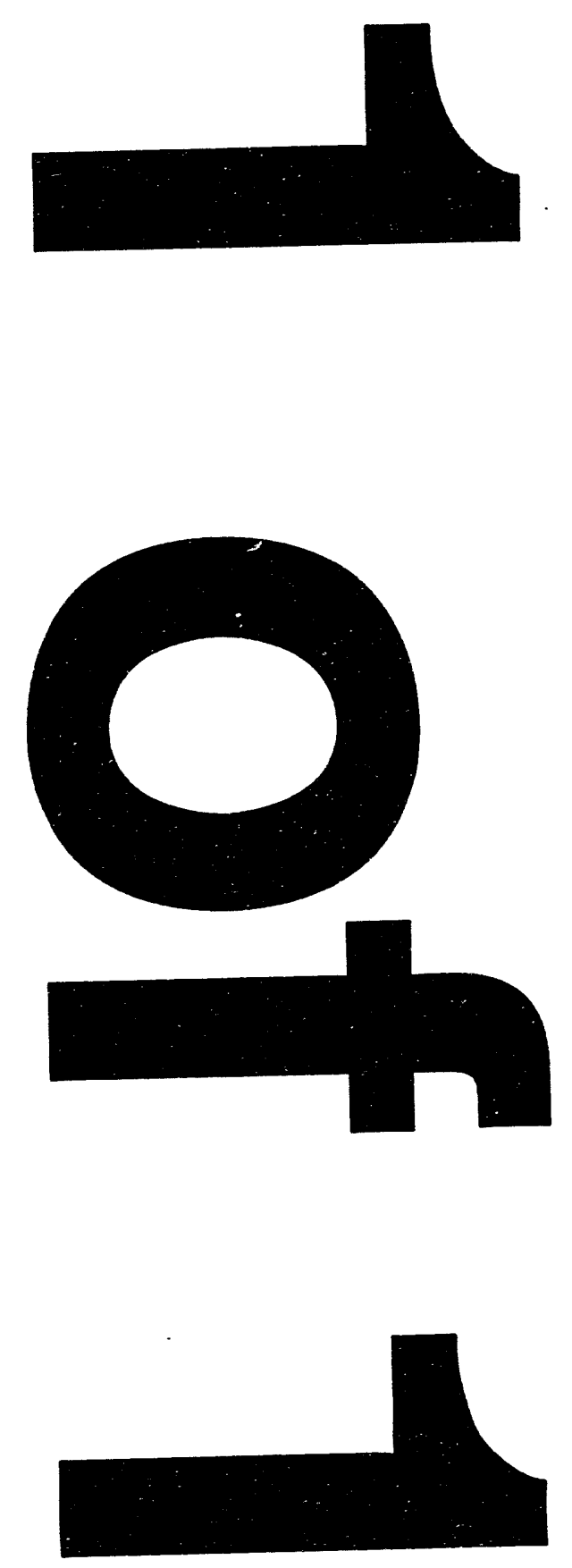


$$
\text { DOE/ER/25/19-2 }
$$

\section{A report to the Department of Energy on Studies of Complexity in Fluid Systems}

Grant DE-FG02-92ER25119

January, 1994

Principal Investigators

All of the PI's are at the University of Chicago, Chicago, IL, 60637.

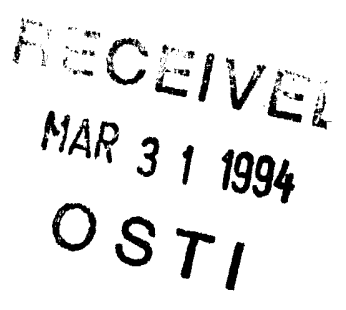

\begin{tabular}{|lll|}
\hline PI & Address & Telephone \\
\hline Peter Constantin & Ryerson Laboratory, 362A & 3127027399 \\
Todd F. Dupont & Ryerson Laboratory, 167 & 3127023485 \\
Leo P. Kadanoff & Research Institutes, L109 & 3127027189 \\
Sidney Nagel & Research Institutes, L115 & 3127027190 \\
\hline
\end{tabular}

\section{Principal Project Personnel}

Peter Constantin, Professor of Mathematics

Role in Project: To analyze the relationships between mathematical properties of the models of fluid flow that arise from other parts of the project.

Principal Areas of Research Expertise: Mathematical aspects of Navier-Stokes turbulence: estimates of bulk dissipation, decay of the power spectrum, scaling exponents.

Percent of Time on Project: About 20\%.

\section{Selected Publications:}

Scaling in fluid turbulence: a geometric theory. P. Constantin and I. Procaccia, Phys Rev. E 47, 3307 (1993).

Energy dissipation in shear driven turbulence, (with $\mathrm{Ch}$. Doering), Phys. Rev. Lett. 69, 1648-1651 (1992).

Global Regularity for Vortex Patches, Commun. in Math. Phys., A. L. Bertozzi and P. Constantin, 152,19-28 (1993).

The dimension of the carrier of dissipation in turbulence: intermittency in fluid mechanics (with I. Procaccia), Phys. Rev. A 46 4736-4741 (1992).

P. Constantin, Ch. Fefferman, Nonlinearity, in press. 
P. Constantin, I. Procaccia, Phys. Rev. E 47, 3307 (1993).

I. Procaccia, P. Constantin, Phys. Rev. Lett., 70, 3416 (1993).

* P. Constantin, I. Procaccia, Nonlinearity, in press.

* P. Constantin, Ch. Fefferman, Indiana Univ. Math. Journal, in press.

* The fractal geometry of the level sets of a contaminant dispersed by chaotic surface waves, P. Constantin and I. Procaccia, Euro. Phys. Lett. 22 (1993).

Droplet Breakup in a Model of the Hele-Shaw Cell P. Constantin, Leo Kadanoff, Todd F. Dupont, R. Goldstein, M. Shelley and S.-M. Zhou. Phys Rev E47, 4169 (1993).

P. Constantin, M. Pugh, Nonlinearity 6 (1993), 393.

* Ch. Doering, P. Constantin, Phys. Rev. E, to appear.

* DoE supported paper

Todd F. Dupont, Professor of Mathematics and Computer Science.

Role in Project:

Construction of software for approximating the solutions of various mathematical models that arise in this study.

Principal Areas of Research Expertise:

Numerical solution of partial differential equations

Percent of Time on Project: About 30\%.

\section{Selecied Publications:}

Mixed finite element methods for time-dependent problems: application to control, with R. Glowinski, W. Kinton, and M. F. Wheeler, Element Analysis in Fluids (T. J. Chung, ed.), 1052-1065, Hemisphere Publishing, (1992).

Explicit/implicit, conservative, Galerkin domain decomposition procedures for parabolic problems, with C. N. Dawson, Math. Comp. 58, 21-34 (1992).

Droplet Breakup in a Model of the Hele-Shaw Cell with P. Constantin, Leo Kadanoff, R. Goldstein, M. Shelley and S.-M. Zhou. Phys Rev E47, 4169 (1993).

* Drop Formation in a One-Dimensional Approximation of the Navier-Stokes Equation, with Jens Eggers, submitted Jour. Fluid Mechanics, October, (1992).

DoE proposal and progress 
* Singularities and Similarities in Fluid Flow, Andrea Bertozzi, Michael Brenner, Todd F. Dupont, and Leo P. Kadanoff, to be published in Springer Verlag Applied Mathematics Centennial Volume.

* Finite-Time Singularity Formation in Hele Shaw Systems, Todd. F. Dupont, Raymond E. Goldstein, Leo P. Kadanoff, and Su-min Zhou, Phys Rev E47 4182 (1993).

* DoE supported paper

Leo P. Kadanoff, John D. and Catherine T. MacArthur Distinguished Service Professor of Physics and Mathematics.

Role in Project: Interpreting experimental results, deriving mathematical models, and conducting numerical studies.

Principal Areas of Research Expertise: Theoretical condensed matter physics.

Percent of Time on Project: About 10\%.

Selected Publications:

Beyond all Orders: Singular Perturbations in a Mapping (with C. Amick, S.C.E. Ching and V. Rom-Kedar), Journal of Nonlinear Science 2, 9 (1992).

Droplet Breakup in a Model of the Hele-Shaw Cell P. Constantin, Leo Kadanoff, Todd F. Dupont, R. Goldstein, M. Shelley and S.-M. Zhou. Phys Rev E47, 4169 (1993).

* Singularities and Similarities in Fluid Flow, Andrea Bertozzi, Michael Brenner, Todd F. Dupont, and Leo P. Kadanoff, to be published in Springer Verlag Applied Mathematics Centennial Volume.

* Finite-Time Singularity Formation in Hele Shaw Systems, Todd. F. Dupont, Raymond E. Goldstein, Leo P. Kadanoff, and Su-min Zhou, Phys Conducting Rev E47 4182 (1993).

* DoE supported paper

Sidney Nagel, Professor of Physics

Role in Project: Experiments in three areas:

Ultrafast Photography of drop snap-off

Flow of granular fluids

Scaling of susceptibility near glass transition 
Principal Areas of Research Expertise:

Condensed matter experimentalist, with experience in studying granular flows and relaxation at liquid/glass transition.

Percent of Time on Project: Approximately $10 \%$.

\section{Publications:}

Instabilities in a Sandpile, S. R. Nagel, Rev. Mod. Phys. 64, 321 (1992).

Physics of the Granular State, H. M. Jaeger and S. R. Nagel, Science 255,1523 (1992).

Sound in Sand, C.-h. Liu and S. R. Nagel, Phys. Rev. Lett. 68, 2301 (1992).

Phase Transitions in a Spring-Block Model of Earthquakes G. Vasconcelos, M. de Sousa Vieira and S. R. Nagel, Physica A 191, 69 (1992).

*Giant Sound-Conductance Changes Caused by Minute Movements of Single Sand Grains, C.-h. Liu and S. R. Nagel (preprint)

Dynamics of Spring-Block Models: Tuning to Criticality, M. de Sousa Vieira, G. Vasconcelos and S. R. Nagel, Phys. Rev. E 47, R2221 (1993).

* Vibration-Induced Size Separation in Granular Media: the Convection Connection, J. B. Knight, H.M. Jaeger and S. R. Nagel, Phys. Rev. Lett. 70, 3728 (1993).

Sound in a Granular Material: Disorder and Nonlinearity, Chu-heng Liu and S. R. Nagel, Phys. Rev. B 48, 15646 (1993).

* Model for Evolution of River Networks, Robert Lehany and Sidney Nagel, Phys. Rev Lett. 71, 1470 (1993).

* DoE supported paper

\section{Additional Faculty Project Personnel}

Robert Almgren was appointed Assistant Professor in the Department of Mathematics in fall 1992. His expertise is in the analysis of models of physical processes, the design and analysis of numerical algorithms, and in computing solutions of systems governed by partial differential equations. In this project he will be studying the solutions of model equations numerically and theoreticaily. 
Andrea Bertozzi is starting her third year as a Dickson Instructor in Mathematics. She has studied the properties of solutions of partial differential equations theoretically and numerically, and that is the role in which she will participate in this project.

Fausto Cattaneo is a Senior Research Associate in the Department of Astronomy. He is accomplished in computational fluid dynamics, particularly the simulation of highly convective flows,.

David Grier is an Assistant Professor in the Physics Department, working in experimental condensed matter physics. He plans to conduct some Hele-Shaw experiments which will be used in studying mathematical models of such situations. His expertise in the use of video image manipulation will play a role in several other experiments as well.

Heinrich Jaeger is an Assistant Professor in Physics. For this project, he works in the experimental study of the flow of granular materials.

Visitors: Itamar Procaccia, Weizmann Institute; Charles Doering, Clarkson.

Postdocs connected to project: Piero Olla, Jonathan Miller, Detlef Lohse, Serge Esipov, Yunson Du, Jens Eggers

Graduate students connected to project: Muhittan Mungan, Jane Wang, Michael Brenner, Elizabeth Grossman, Robert Lehany, Mary Pugh

\section{Project Overview:}

The Objective of this project is to bring together researchers from several disciplines who share an interest in studying the development of complexity in fluid systems. The researchers involved have expertise in mathematics, numerical computation, and theoretical and experimental physics.

There are three main subjects:

The mathematical and phenomenological approaches to turbulence. item

The development of singularities in fluid flow, most particularly in the motion of interfaces. Mathematical and numerical studies of viscous droplet generation.

Studies of the scaling properties of highly viscous liquids and the nature of avalanche dynamics in granular flows. Connections 
between the experiments and simulation have been particularly emphasized.

In each case, we are trying to look at a simple, but nontrivial, experimental situation; build a simplified mathematical model; and study the properties of the model theoretically and numerically. In turbulence the approach is primarily analytic in nature: use the PDE. In the area of physics of granular systems the approach has been experimental, using actual sand piles and idealized models.

We are concerned with obtaining a better understanding of how complexity manifests itself in the world around us, and thereby perhaps better understanding of the degree of predictability in some specific situations. Our main objects of study will be fluid and granular materials, but we hope that the physical and mathematical methods which we develop might have an even broader applicability. In addition, we hope to train a number of students and postdocs who will be able to work on interdisciplinary projects on the borderline between math and physics.

\section{Previous Work:}

This project started on 1 May, 1992. However, the project carried forward considerable earlier work in the area, as can be seen form the lists of selected publications given above. For example, in the area of turbulence we built upon the work of Constantin, Kadanoff and others on transitions in convective turbulence; the results of Constantin and Doering on energy dissipation in turbulence; and the fractal isoscalar surface paper of Constantin, Procaccia and Sreenivasan. For granular materials, we started from studies in which we study the effect of shaking on the spontaneous demixing of a granular material according to the size of the particles. It is often, but not always, found that larger particles tend to rise in a sea of smaller ones when the whole system is shaken. In fluid flow singularities, we begun from the study of droplet breakup in HeleShaw cells, carried out by several of the people listed here and the work on drop formation by Dupont and Eggers.

\section{Completed Work}

1. Giant Sound-Conductance Changes Caused by Minute Movements of Single Sand Grains Chu-heng Liu and Sidney R. Nagel have investigated how a small disturbance of a sirigle grain of sand can affect the transmission of sound in an unconsolidated granular material. They found that thermal expansion of a single grain due to 
a $1 \mathrm{~K}$ temperature rise $(\sim 2000 \mathrm{~A})$ can significantly change the transmission even though this expansion is minute compared to either the grain diameter $(0.5 \mathrm{~cm})$ or the sound wavelength $(\sim 1 \mathrm{~cm})$. The extreme sensitivity is unique to sand and diffusive-wave theory cannot account for this behavior without including further postulates about the nature of the scattering centers. By installing 50 heaters inside a sand pile we have also investigated the spatial pattern of the wave propagation. The disturbance due to the heaters is very irregular in space: two adjacent heaters can give very different responses. This suggests that the medium is very inhomogeneous. The average over many configurations of the rms disturbance decays with distance from the source or detector.

2. Droplet Simulations in axial symmetry. Eggers and Dupont have set up a set of lubrication equations for the motion of a viscous fluid with surface tension. The simulational result from these equations compare very well with experimental observations of flows of jets and water drops.

\section{Proposed Work}

1. Cascade models: Mogens Jensen and Roberto Benzi have very effectively exploited a cascade model of turbulence. We have imf oved the program for the model and are starting to use it. Our ur will include: the calculation of time correlation functions, an atcempt to reproduce the known enhancement of the spectrum at the high-k end of the inertial range, and an attempt to see what the effect of one branch in the cascade might produce.

We are also trying to analyze the model to see the extent to which it is like a Hamiltonian system and whether there are other nearby models which are integrable.

2. Singularity Structure in Model Fluid Interfaces. These form singularities as masses of fluids separate from one another. With experiments, we have looked at how the singularity occurs as the viscosity of the liquid is varied. As the viscosity increases it appears that the form of the singularity changes shape. This is a collaboration of X. D. Shi who has been doing the experimental work, Mike Brenner who has been involved in the simulations and analytic theory and Todd Dupont and Jens Eggers who have developed the initial code for the simulations, and David Grier who has worked on the two dimensional problem.

Michael Brenner and the experimental group are looking at the nature of the necking instability in axially symmetrical fluids. Is the 
Eggers single isolated neck ever stable? Why? or Why not? Is the multiple necking solution the universal end result?

Kadanoff, Dupont, Bertozzi, Grossman, and Brenner are moving on from the old work on similarity solutions to studying bifurcations in the behavior of similarity solutions to

(1) $h_{t}+\left(h^{n} h_{x x x}\right)_{x}=0$

We do not understand the behavior in the neighborhood of any of the bifurcations in this equation. The simplest case is $n=2$ bifurcation in the infinite time singularity to equation (1). We want to know the nature of the singularities in Eq (1) for pressure boundary conditions as $t$ goes to infinity and $\varepsilon=n-2$ goes to zero.

3. Energy dissipation in turbulent flow. The problem of estimating the energy dissipation in shear turbulent flows has received a new treatment leading to variational problems with spectral constraints ([1], [14]). The estimates of ([1]) were in good agreement with the data from the Taylor - Couette turbulence experiment of Lathrop, Fineberg and Swinney even before the variational problem was solved. We have solved now the first such variational problem. We are planning to continue our efforts with the aim of improving logarithmically (if possible) our bound. Other applications of the method will be to estimate energy dissipation in convective turbulence and in pipe flow. The work is done in collaboration with Ch. Doering who visited the University of Chicago in the Fall Quarter, 1993.

4. Models for incompressible generation of small scales. In collaboration with A. Majda and E. Tabak from Princeton we study active scalars ([2]) which are theoretical two dimensional models of three dimensional incompressible fluid flows, but are also interesting on their own as physical models - especially in geophysics. We propose to investigate the possibility of finite time singularities and the depletion of curvature as predicted theoretically and numerically ([3], [4]).

5. Scaling exponents in turbulence. We will continue our study ([5]) of the generalized structure functions of velocity in turbulent flow. We intend to make contact between the analytic ([6]) aspects of the theory and the geometric ([7], [8], [9]) ones. Our collaborators are Ch. Fefferman (Princeton) and I. Procaccia (Weizmann).

6. Singularities in incompressible fluids. We intend to pursue the investigations of ([2], [3]) regarding the role of the direction of vorticity in singularity formation. The possibility of viscous 
suppression of singularities ([10]) and the possibility ([11]) of inviscid singularities will be investigated analytically and numerically. Collaborators are R.M. Kerr (NCAR), A. Majda, Ch. Fefferman.

7. Motion of interfaces. We will consider the Hele--Shaw singularity problem ([12], [13]) in a real variables formulation. Collaborators are T. Dupont and L.P. Kadanoff.

8. Size segregation in vibrated granular mixtures. This segregation is typically considered a phenomenon separate from vibrationally induced convection. Nagel, Jaeger, and Knight [15] have presented the first experimental evidence that in some systems segregation arises from convective processes. They investigated the rise of a single large glass bead through a column of smaller glass beads. This mixture was confined to a cylindrical container and subjected to discrete, vertical excitations. A cylindrically symmetric convection cycle, rising in the middle of the column and dropping along the walls of the container, was found to be responsible for all particle movement. Particles larger than the width of the downward convection zone are carried to the top of the column and then trapped, resulting in size 'segregation'. We shall continue to try to develop models which explain the motion, particularly the effect of the walls.

In addition, we wish to explore the surface behavior of highly agitated (vibrated) granular materials. In this limit we approach a state of matter that might be termed "granular fluids". These fluids have an effective viscosity but no surface tension. Yet they do exhibit many phenomena known from ordinary hydrodynamics. One particularly interesting aspect: in this limit there are parametrically induced Faraday instabilities (at half the drive frequency), just as in liquids where surface tension is known to be important for the correct description.

9. River patterns. Lehany and Nagel [16] have developed a model, which includes the effects of erosion both from precipitation and from avalanching of soil on steep slopes, to simulate the formation and evolution of river networks. The avalanches provide a mechanism for competition in growth between neighboring river basins. The changing morphology follows many of the characteristics of evolution set forth by Glock. We find that during evolution the model maintains the statistical characteristics measured in natural river systems. 


\section{Bibliography of DOE-supported papers}

Scaling in fluid turbulence: a geometric theory, P. Constantin and I. Procaccia, submitted to Physics of Fluids, September, 1992.

Drop Formation in a One-Dimensional Approximation of the NavierStokes Equation, with Jens Eggers, submitted Jour. Fluid Mechanics, October, 1992.

Finite-Time Singularity Formation in Hele Shaw Systems, Todd. F. Dupont, Raymond E. Goldstein,. Leo P. Kadanoff, and Su-min Zhou, Phys Rev E47 4182 (1993).

P. Constantin, Ch. Fefferman, Indiana Univ. Math. Journal, in press.

Vibration-Induced Size Separation in Granular Media: the Convection Connection", J. B. Knight, H.M. Jaeger and Sidney R. Nagel, Phys. Rev. Lett. 70, 3728 (1993).

Giant Sound-Conductance Changes Caused by Minute Movements of Single Sand Grains, Chu-heng Liu and Sidney R. Nagel, preprint.

Bifurcation of Falling Liquid Droplets, X. D. Shi, D. G. Grier and Sidney R. Nagel, preprint.

Singularities and Similarities in Fluid Flow, Andrea Bertozzi, Michael Brenner, Todd F. Dupont, and Leo P. Kadanoff, to be published in Springer Verlag Applied Mathematics Centennial Volume.

The fractal geometry of the level sets of a contaminant dispersed by chaotic surface waves, P. Constantin and I. Procaccia, Euro. Phys. Lett. 22 (1993).

Non-Kolmogorov scaling exponents and the geometry of high Reynolds number turbulence, Peter Constantin and I. Procaccia, submitted

Scale Resolved Intermittency in Turbulence, Siegfried Grossmann and Detlef Lohse, preprint. 


\title{
REFERENCES
}

[1] Ch. R. Doering and P. Constantin, Phys. Rev. Lett 69, 1648, (1992).

[2] P. Constantin, Arg. Natl. Lab. preprint ANL/MCS-TM-170, (1992).

[3] P. Constantin, SIAM Review, to appear.

[4] P. Constantin, A. Majda, E. Tabak, Phys. Lett. A, in press

[5] P. Constantin, Univ. Minn. AHPCRC preprint 92-096, (1992).

[6] P. Constantin, Ch. Fefferman, Nonlinearity, in press.

[7] P. Constantin, I. Procaccia, Phys. Rev. E47, 5 (1993), 3307

[8] I. Procaccia, P. Constantin, Phys. Rev. Lett., 70, 3416, (1993).

[9] P. Constantin, I. Procaccia, Nonlinearity, in press.

[10] P. Constantin, Ch. Fefferman, Indiana Univ. Math. Journal, in press.

[11] R.M. Kerr, submitted Phys. Fluids A, Nov.. 1992.

[12] P. Constantin, Leo Kadanoff, Todd F. Dupont, R. Goldstein, M. Shelley and S.-M. Zhou. Phys Rev E47, 4169 (1993).

[13] P. Constantin, M. Pugh, Nonlinearity, 6, (1993), 393.

[14] Ch. Doering, P. Constantin, Phys. Rev. E, to appear.

[15], J. B. Knight, H.M. Jaeger and Sidney R. Nagel, Phys. Rev. Lett. 70, 3728 (1993).

[16] Robert Lehany and Sidney Nagel, Phys. Rev Lett. 71, 1470 (1993).

\section{DISCLAIMER}

\begin{abstract}
This report was prepared as an account of work sponsored by an agency of the United States Government. Neither the United States Government nor any agency thereof, nor any of their employees, makes any warranty, express or implied, or assumes any legal liability or responsibility for the accuracy, completeness, or usefulness of any information, apparatus, product, or process disclosed, or represents that its use would not infringe privately owned rights. Reference herein to any specific commercial product, process, or service by trade name, trademark, manufacturer, or otherwise does not necessarily constitute or imply its endorsement, recommendation, or favoring by the United States Government or any agency thereof. The views and opinions of authors expressed herein do not necessarily state or reflect those of the United States Governmeist or any agency thereof.
\end{abstract}


11
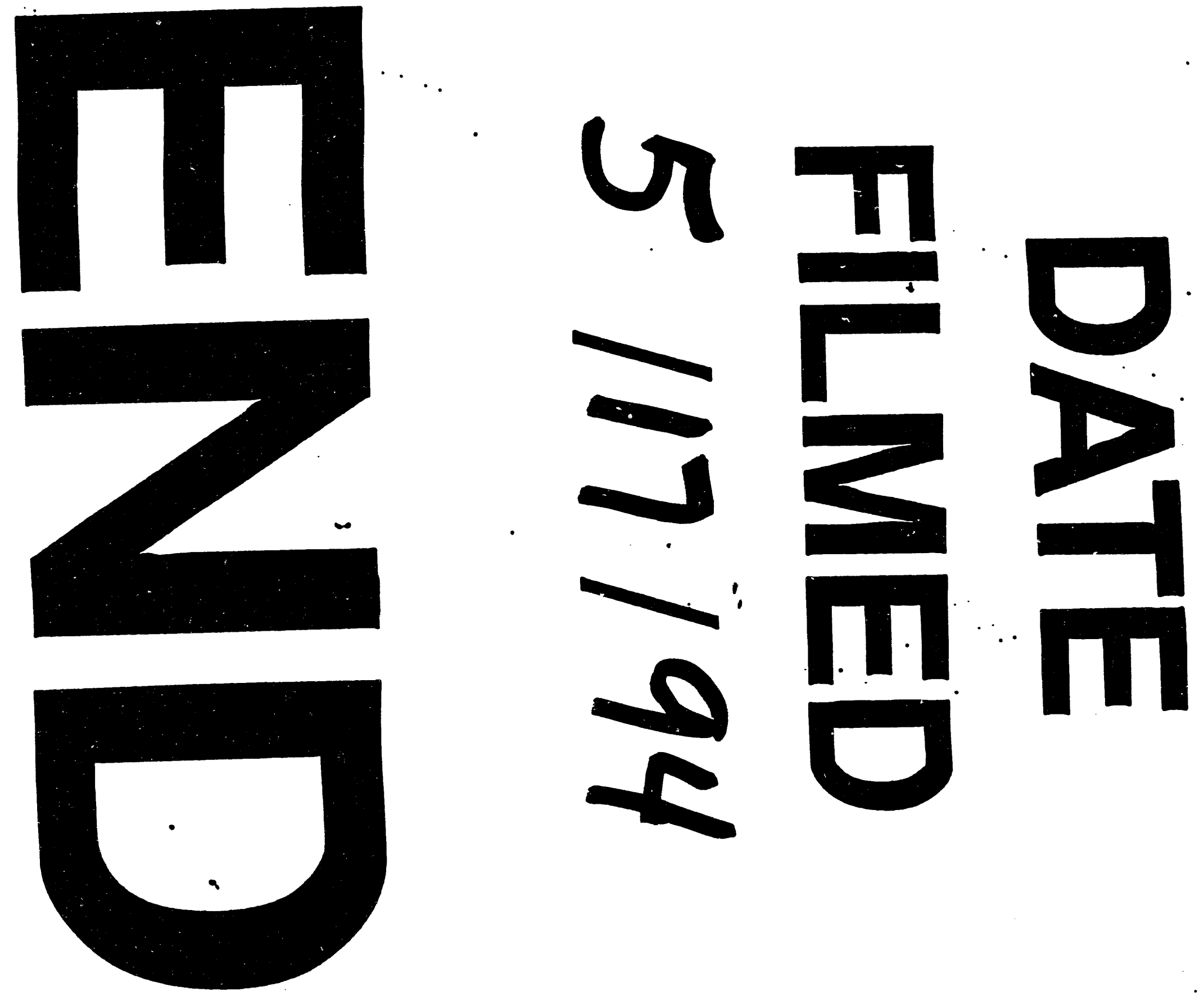
